\title{
PERCHÉ LE POLITICHE CAMBIANO: \\ IL CASO DEI TRASPORTI
}

\author{
di Mauro Tebaldi
}

\section{Introduzione}

Il tema del policy change è da tempo al centro di un articolato dibattito intorno al quale si confrontano gli argomenti di chi, come l'autore di queste note, ritiene che il problema vada ben inquadrato in sede teorica avanti qualsivoglia indagine sui processi evolutivi delle politiche pubbliche. Sebbene assai poco frequentato dai cultori della scienza politica, il settore delle politiche dei trasporti richiama un campo di attività pubblica oltremodo rilevante, la cui esplorazione sistematica, alla luce degli studi sinora condotti, solleva questioni di ordine teorico e metodologico particolarmente salienti per l'analisi comparativa del policy change.

I percorsi evolutivi delle politiche dei trasporti e, con essi, le loro dinamiche di mutamento, vengono infatti spiegati, in misura prevalente, attraverso fattori di natura esogeno-ambientale (le macro variabili economiche e politico-istituzionali) oppure facendo ricorso al paradigma del decisore razionale e auto-interessato, altresì alieno dalle eredità storiche e dai mutevoli vincoli ideologici che spesso caratterizzano le scelte, o non-scelte, di policy. Come spiegare, tuttavia, sulla scorta di questi modelli, i profondi mutamenti che negli ultimi venti anni hanno trasformato orientamenti generali, obiettivi e strumenti delle politiche dei trasporti in gran parte delle democrazie euro-atlantiche, indipendentemente dalla successione dei cicli economici o dalle modificazioni intervenute a livello di politics? E perché politiche dei trasporti inefficienti sia sotto il profilo del benessere sociale, sia in base ai calcoli di utilità dei soggetti-decisori, persistono nel tempo secondo derive inerziali tendenzialmente impermeabili a interventi innovativi, quando non a programmi di riforma globale? Perché, infine, cambiamenti anche significativi 
nella struttura della policy vengono talvolta introdotti malgrado non sussista alcuna domanda sociale a ciò indirizzata, né un evidente deficit decisionale che ne renda necessaria la sua trasformazione?

I (pochi) contributi più specificamente orientati all'analisi del policy making settoriale non offrono risposte esaustive a questi interrogativi. Essi non sempre si sottraggono alla semplice, ma erronea, equazione «stabilità del policy network = stabilità (e tendenziale irreversibilità) della politica pubblica». E anche laddove l'ipotesi viene revocata in dubbio, $i$ casi in esame testimoniano che assetti stabilizzati delle configurazioni decisionali danno sì luogo a mutamenti non occasionali delle politiche adottate, ma limitatamente a strumenti, tattiche e interventi mirati: non a quei cambiamenti paradigmatici che sollevano i maggiori problemi interpretativi e che costituirebbero il frutto, secondo questi autori, di pressioni essenzialmente esogene al reticolo decisionale.

Nell'ambito della letteratura presa in esame sfuggono, pertanto, quelle dinamiche tipicamente endogene senza la cui comprensione risulta debole qualunque teoria del mutamento. Detto altrimenti, non sembra sufficientemente evidenziato, o quantomeno resta confinato al rango di variabile interveniente, l'insieme dei fattori morfologico-organizzativi e autoritativo-relazionali che opera all'interno del campo di policy e che agisce quali autonomo veicolo di sviluppo della politica pubblica. $\mathrm{Ci}$ avvarremo del concetto di istituzionalizzazione per dar conto di entrambi gli aspetti della politica dei trasporti, ossia per esplicitare le specifiche modalità con cui tale politica assume forma organizzativa e forza decisionale indipendentemente da variabili esogene o da strategie di massimizzazione dell'utilità attesa. A questo proposito, sosterremo l'ipotesi che il grado di istituzionalizzazione della politica dei trasporti agisca quale fattore di sviluppo endogeno del campo organizzativo, capace di generare dinamiche di policy change alimentando strategie di protezione e difesa rispetto ai potenziali elementi di discontinuità ambientale, siano essi tecnologici, economici o politico-istituzionali. E ciò non solo in base ad una logica conservativa degli equilibri e delle risorse di potere all'interno della coalizione dominante, ma anche e soprattutto grazie alla capacità di adattamento dinamico e responsiveness agli stimoli esterni che l'istituzionalizzazione consente. Una sintetica analisi comparata, estesa a cinque democrazie dell'area euro-atlantica (Regno Unito, Francia, Sve- 
zia, Italia, Stati Uniti), consentirà infine di sondare la portata, l'estensione e la forza esplicativa di tale ipotesi.

Beni pubblici, pubbliche utilità e scelta razionale nel mercato dei trasporti

Parafrasando una nota definizione di Dunn (1981), il campo di analisi della politica dei trasporti può essere esteso all'insieme di azioni e comportamenti correlati (sia positivamente che negativamente) al «trattamento» di un problema di mobilità nello spazio, ossia alla risposta a una domanda, un bisogno o un'opportunità insoddisfatta di intervento pubblico inerente lo spostamento di persone o cose su un territorio. Al centro della definizione è collocato il concetto di «mobilità fisica», che evoca uno dei bisogni prioritari espressi dalle società modernizzate nel corso dei processi di sviluppo dell'ultimo secolo. Elementi e ipotesi di correlazione fra evoluzione dei sistemi di trasporto e modificazioni di stato dei sistemi politici, economici e sociali sono ampiamente disponibili in letteratura, laddove il tema della mobilità costituisce specifico oggetto di studio sia come explanans, che come explanandum delle developmental theories.

Il problema delle politiche della mobilità come fenomeno da spiegare - dal quale prendiamo le mosse nel presente lavoro - è stato affrontato seguendo diverse prospettive di ricerca, con centralità più o meno accentuata. Si possono distinguere tuttavia due filoni di analisi principali, uno di natura micro-economica e l'altro di impostazione macro-storica, entrambi articolati, al loro interno, in correnti teoriche anche fortemente differenziate. La prima direttrice di ricerca, di carattere micro-economico, si concentra sul tipo e grado di razionalità espressi dall'intervento pubblico. Intende cioè verificare se e a quali condizioni le risposte al problema della mobilità fornite dalle politiche di governo risultano prevalentemente congruenti a principi di efficacia, efficienza o equità. Le acquisizioni teorico-normative della economia del benessere applicate ai trasporti evidenziano pertanto quando è preferibile l'intervento della mano pubblica in alternativa o in aggiunta al mercato e in quale misura la regolazione statale può strutturare arene e processi «virtuosi» in grado di ottimizzare l'allocazione di risorse scarse. Le scienze economiche offrono consolidati strumenti teorici per comprendere, sotto il profilo prescrittivo, perché il perseguimento del benessere col- 
lettivo implichi il passaggio dal mercato allo stato e quando invece risulti conveniente percorrere la strada inversa. La razionalità dell'intervento pubblico è imperniata non solo e non tanto sulla necessità di anticipare e incentivare l'ingresso nel mercato di capitali sufficienti a costituire una «offerta» di trasporto; essa discende, soprattutto, dal bisogno di indirizzare e sostenere la «domanda» di mobilità, al fine di eliminare, o quantomeno correggere, le condizioni di «fallimento del mercato» 1 .

Sia nel caso del trasporto collettivo, sia in quello del trasporto individuale, l'approccio economico-normativo identifica i percorsi e i contenuti decisionali di massima utilità per la collettività di riferimento, date una certa configurazione delle risorse disponibili e una determinata funzione del benessere sociale, inteso come sommatoria delle funzioni di preferenza individuali. L'ottima strategia - quella che massimizza i vantaggi sociali - consente di stabilire quando e in quale misura lo stato dovrebbe sostituirsi al mercato come arena per l'allocazione dei beni. Se, tuttavia, tali assunzioni di razionalità ci indicano quali alternative ottimizzanti sono a disposizione del decisore per correggere le imperfezioni del mercato, rispetto a un ipotetico calcolo di utilità collettiva, poco o nulla dicono circa la persistenza e la riproduzione di soluzioni sub-ottimali - quando non esplicitamente svantaggiose - nel computo delle risorse pubbliche in gioco. Detto in altri termini, se la scelta pubblica nei trasporti dovrebbe essere mirata a scongiurare il fallimento del mercato e a riequilibrare la distribuzione dei costi e benefici sociali, solo in rari casi le politiche sembrano esprimere con chiarezza tali finalità e i principi di razionalità decisionale ai quali si ispirano. Anzi, anche restando all'interno degli approcci microeconomici, l'evidenza empirica sembra rafforzare ipotesi regolative in cui, piuttosto che il benessere pubblico, prevalgono gli

1 Più specificamente, la regolazione, il finanziamento, la realizzazione di investimenti e, quando necessario, la gestione diretta del servizio da parte dello stato, perseguono tre obiettivi di equilibrio sociale. Innanzitutto, quello di impedire la formazione di monopoli privati in un settore ritenuto strategico dalla comunità economica e politica; in secondo luogo, l'intervento statale assume una valenza limitativa o preclusiva agli investimenti privati, intendendo sottrarre ad un uso speculativo servizi di interesse generale, la cui larga accessibilità determina l'efficacia delle politiche perseguite; la necessità di interventi regolativi è in terzo luogo indotta dalla allocazione imperfetta dei diritti di proprietà, talché la produzione concentrata di esternalità risulta disgiunta dalla diffusione dei costi: lo stato può intervenire autoritativamente allocando in modo selettivo tali diritti o facendoli propri, assieme alle responsabilità e agli oneri degli spill-over (Button 1990). 
interessi di specifici attori: i soggetti sottoposti o da sottoporre alla regolazione secondo le teorie della cattura (Glaister e Mulley 1983); le funzioni di utilità dei decisori - siano essi politici o burocrati - secondo le teorie della public choice (Stigler 1971; Mc Bride 1990).

Da questi contributi ${ }^{2}$ emerge, in particolare, che i criteri puramente economici - di riduzione dei costi e massimizzazione dell'efficienza, di miglioramento della qualità del servizio, ecc. sono raramente adottati dai decisori politici e amministrativi come prioritari parametri di scelta, e difficilmente, di per sé, riescono a generare decisioni significative, se non in presenza di vincoli di budget ineludibili. Questo perché il rapporto con il proprietario che delega politici e burocrati ad amministrare il bene, cioè la collettività dei contribuenti/elettori, è avvertito in modo lontano e distorto, e perché il miglioramento economico della gestione non comporta, sic et simpliciter, benefici e riconoscimenti apprezzabili per i responsabili pubblici (Fokkema e Nijkamp 1994). Di conseguenza, le decisioni in materia di servizi e infrastrutture di trasporto sono influenzate, oltreché dalla razionalità economica della allocazione efficiente, da fattori di convenienza eminentemente politica, o comunque di carattere non monetario: la consistenza e il grado di organizzazione dei gruppi sociali e dei gruppi di interesse dei quali il decisore pubblico è rappresentante, o ai quali fa riferimento come sostenitori elettorali; la presenza e il peso di altri decisori pubblici, alleati, concorrenti o avversari, in merito alle decisioni da prendere; le risorse finanziarie pubbliche di cui può disporre in relazione alla decisione specifica e alle altre da inserire in agenda; le risorse umane e organizzative di cui dispone per l'attuazione della scelta.

2 Nell'ambito della public choice, sono numerose le analisi di decisioni pubbliche concernenti direttamente o indirettamente i trasporti collettivi. Esse riguardano principalmente quattro aree tematiche: la determinazione della natura di bene pubblico o privato del trasporto collettivo; i criteri che guidano la scelta del decisore pubblico fra produzione pubblica e produzione privata di servizi finanziati dalla collettività, come quelli di trasporto (Mc Guire et al. 1987); il rapporto tra scala dimensionale dell'amministrazione pubblica ed efficienza del servizio di trasporto pubblico (Dilorenzo 1983); gli effetti di redistribuzione di costi e benefici tra gruppi di interesse derivanti da scelte di nazionalizzazione/privatizzazione di industrie e servizi (Pint 1990). 
Modernizzazione, sviluppo e dinamiche dell'intervento pubblico per la mobilità

Postulando l'esistenza di un grado di correlazione fra sviluppo politico, sociale, economico e variazioni nella domanda e nell'offerta di mobilità, l'insorgenza, il consolidamento e l'eventuale degenerazione di una politica pubblica dei trasporti dipendono pertanto, oltre che dalla ricerca di obiettivi ottimo-paretiani, dalle modalità con cui la correlazione si è strutturata $\mathrm{e}$ si è andata modificando nel tempo in ciascun contesto nazionale. Come, tuttavia, all'interno del legame causale fra domanda di mobilità e fattori socio-economici di modernizzazione, influiscono o possono influire le variabili politico-istituzionali nel rafforzare, indebolire o, al limite, annullare tale nesso, modificando altresì le forze del mercato? In altri termini, come interferiscono e quanto pesano le dinamiche fra attori, arene e istituzioni politiche nella determinazione della spesa e della regolamentazione settoriale, posta la natura pubblica o semi-pubblica delle infrastrutture e di gran parte dei servizi di trasporto collettivo? L'attuale e la passata configurazione della domanda e dell'offerta di mobilità, ai diversi livelli territoriali, quanto riflette le mutate condizioni socio-economiche o quanto, piuttosto, è indotta dai differenti percorsi di sviluppo politico-istituzionale sperimentati dai paesi oggetto di studio?

Il problema è stato analizzato secondo una prospettiva di ricerca di carattere macro-storico, per la quale la politica pubblica è un prodotto di forze sistemiche fra loro interagenti. Nell'ambito di questa direttrice comune, si possono individuare due prospettive teoriche antagoniste: un modello privilegia i fattori esplicativi di natura socio-economica e riduce quelli politici al rango di variabile interveniente; l'altro è imperniato sulla preminenza dei processi politici di state e nation building prima, e di democratizzazione poi, quali fattori esplicativi dei tempi, dei modi e della consistenza dell'intervento pubblico. Sotto il profilo longitudinale, le trasformazioni e le differenze nelle modalità di intervento settoriale dei governi variano in funzione dei processi di industrializzazione e di accumulo del capitale, e ai fenomeni di mobilitazione sociale a cui tali processi danno luogo. Secondo questo schema interpretativo, le logiche dello sviluppo industriale avrebbero condotto alla convergenza e all'allineamento progressivo dei regimi liberal-democratici lungo direttrici di crescita del settore pubblico dai carat- 
teri similari. L'ipotesi di fondo è che ciascuna fase di sviluppo dell'economia moderna abbia generato classi differenziate di funzioni pubbliche, in cui prevalgono specifici indirizzi di governo e obiettivi di spesa. Ad un trend di aumento generalizzato della spesa pubblica non corrisponde, pertanto, una ripartizione settoriale secondo criteri di proporzionalità. Si assiste piuttosto a un processo di scostamento fra le opzioni di spesa, con una accelerazione delle prestazioni sociali e un declino degli esborsi per dotazioni infrastrutturali, via via che si passa dalla industrializzazione matura ai modelli di produzione postfordista ${ }^{3}$.

Di converso, all'interno del modello geo-politico formulato da Stein Rokkan (1982), l'intervento statale volto alla realizzazione di infrastrutture di trasporto rientra fra i requisiti funzionali allo sviluppo e al mantenimento dei sistemi politico-territoriali: le reti infrastrutturali, insieme alle agenzie che le amministrano, costituiscono parte delle strutture istituzionalmente adibite al controllo della produzione e distribuzione secondaria e terziaria; sono altresì alcune fra le principali forme organizzative impiegate per la protezione dei confini e dell'ordine interno da eserciti ed apparati repressivi. Si tratta, dunque, di requisiti struttural-funzionali generati, rafforzati o sottodimensionati in precise fasi temporali, scandite da momenti di crisi di sviluppo dei moderni stati nazionali. La nascita delle reti infrastrutturali di trasporto si inserisce nella composita gamma di risposte fornite dalle élite politiche di governo di fronte alle sfide di penetrazione, integrazione, partecipazione e distribuzione.

La crisi di penetrazione concerne l'estensione del controllo del centro sulla periferia. Essa viene affrontata con soluzioni istituzionali mirate alla creazione di una amministrazione centralizzata sul territorio nazionale. Cruciale, a questo riguardo,

3 Le categorie dello sviluppo economico elaborate da Rostow (1971), individuano quattro fasi fondamentali a cui si associano, nelle moderne democrazie di massa, configurazioni peculiari dei rapporti di potere fra i soggetti istituzionali e le forze sociali (partiti, gruppi, movimenti) progressivamente mobilitate dalla apertura dei canali partecipativi, dalla estensione dei diritti civili e dalla crescita delle aspettative di benessere. In concomitanza all'aumento di ricchezza prodotta e alla diffusione sociale del potere, si verifica un incremento dell'intervento pubblico nella fornitura di quei beni e servizi atti a soddisfare le domande dei cittadini. Il rapporto fra tasso di crescita economico-industriale, livello di aggregazione/articolazione delle domande e responsiveness dei governi genera pertanto la quota e il mix di spesa pubblica nelle differenti fasi di sviluppo dei regimi poliarchici. 
risulta l'esistenza delle reti infrastrutturali necessarie alla mobilitazione nello spazio delle risorse economiche (imposizione fiscale, mano d'opera, materie prime) e di violenza (eserciti, armamenti), oltreché alla instaurazione e al mantenimento dell'ordine pubblico. Dalle risposte alla sfida di integrazione emergono le regole allocative delle risorse tra i settori territoriali, culturali, etnici facenti parte della comunità nazionale. Il livello di equità distributiva tra i segmenti, e quindi il grado di integrazione nazionale perseguita, concorre a determinare la quota del bilancio pubblico assegnata agli enti periferici o regionali per investimenti in infrastrutture stradali e ferroviarie. Problemi quali la gestione, il finanziamento e la regolazione dei trasporti estendono tuttavia la loro rilevanza politica, fino a collocarsi fra le issues fondamentali delle poliarchie occidentali, allorché la liberalizzazione e la partecipazione consentono di stabilire un nesso significativo tra le domande dei cittadini e le risposte dei governanti, mediante l'espressione del consenso e la difesa dei diritti civili. Cioè quando, in risposta alla sfida di partecipazione, vengono fissate regole, istituzioni e soggetti della rappresentanza democratica. Il processo di estensione del suffragio costituisce una formidabile chiave di ingresso nel circuito decisionale degli strati non privilegiati della comunità nazionale: allorché esso si compie, i temi della politica entrano in rapporto diretto con i cittadini, mobilitati attraverso gli strumenti della partecipazione e le garanzie di contestazione pubblica. L'apertura dei canali rappresentativi implica inoltre lo sviluppo dei moderni partiti di massa, che dispongono dei filtri ideologici e degli orientamenti valoriali a cui collegare le scelte di policy. L'opzione fra trasporto pubblico e trasporto privato, la scelta fra politiche della mobilità incentrate sull'equità oppure sull'efficienza allocativa, l'esercizio dei sistemi di trasporto secondo gli equilibri del mercato o la loro regolamentazione entro rigidi vincoli programmatici, diventano così questioni fortemente interrelate alle dinamiche del consenso, articolate, mediate e incanalate dai partiti politici e dalle organizzazioni degli interessi ${ }^{4}$.

4 Attori, contenuti e regole del gioco del policy field sono infine fortemente influenzati dagli esiti della sfida di distribuzione, relativa alle modalità di intervento dello stato nella riallocazione delle ricchezze prodotte fra i diversi strati della cittadinanza; intervento che nel settore dei trasporti pubblici assume generalmente duplice carattere: sia diretto, sotto forma di finanziamenti per infrastrutture e sussidi per ripianare il deficit di esercizio del gestore pubblico, sia indiretto, tramite il rafforzamento delle oppor- 
Fra politics e policy: influenze esogene e fattori endogeni

Ritorniamo al quesito di partenza. Perché la mobilità diventa un problema collettivo e si afferma come autonomo settore di policy, ossia quali motivazioni orientano l'intervento pubblico e quali condizionamenti ne stabiliscono l'estensione? Quali sono, soprattutto, le variabili più rilevanti nel determinare la tendenza alla continuità e al cambiamento delle politiche dei trasporti? Come ci hanno indicato le teorie micro-economiche, la mobilità delle persone e delle merci implica l'intervento pubblico nella regolamentazione del mercato, nella realizzazione delle infrastrutture di trasporto e, talvolta, nel loro esercizio. Sia che assumano i caratteri di beni pubblici, sia che rientrino nella categoria delle pubbliche utilità, i beni e i servizi di trasporto recano molteplici cause di fallimento del mercato (monopoli, esternalità, free riding, ecc.), creando uno «spazio naturale» per l'azione amministrativa e regolativa dei pubblici poteri. In base a variabili esplicative di ordine macro-economico, l'intervento si è storicamente manifestato allorché il decisore pubblico, sotto la pressione della crescita industriale e tecnologica, ha cercato di instaurare le condizioni di funzionamento del mercato, anticipando e incentivando l'ingresso dei capitali necessari alla costituzione di una offerta di trasporto. Il significato e la portata del controllo pubblico si sono quindi modificati al mutare dei fattori di modernizzazione, ove è prevalso l'imperativo di correggere le distorsioni del mercato mediante una gestione diretta e di operare efficientemente in regime di effettiva concorrenza, per evitare ritardi e strozzature ai percorsi di crescita socio-economica. D'altro lato, le dinamiche dello sviluppo politico hanno influenzato in modo altrettanto evidente i processi evolutivi delle reti di comunicazione e le relative politiche di intervento: modellando le arene, le regole e gli stili del policy making.

tunità e delle facilitazioni a disposizione dei gruppi sotto-privilegiati. È dunque evidente che l'imposizione di oneri redistributivi tali da consentire una offerta pubblica dei servizi di trasporto e l'erogazione delle risorse finanziarie per la fornitura diretta del trasporto collettivo tendano ad ingigantire il processo di cumulazione delle attività e delle responsabilità governative. Al punto che le amministrazioni delle odierne democrazie di massa vedono estendere il proprio spettro di azioni dall'investimento per la creazione di veri e propri beni pubblici (strade, ferrovie, porti, aeroporti, ecc.), alla regolazione dell'uso di tali beni, alla ripartizione dei costi delle esternalità, fino alla gestione semi-monopolistica dei sistemi di trasporto collettivo, in concorrenza con i modi di trasporto privato. 
Le teorie esaminate forniscono tuttavia risposte parziali alle nostre esigenze conoscitive. I modelli micro-economici concepiscono il decisore come un soggetto a-storico, la cui razionalità non tiene conto delle esperienze pregresse e dei vincoli valoriali se non come dati costanti e immutabili (Etzioni 1990), finendo così per identificare la politica pubblica con l'insieme delle scelte che massimizzano l'utilità di categorie circoscritte o della società nel suo complesso. Quelli imperniati sulle variabili dello sviluppo economico affrontano il tema dell'insorgenza pubblica del problema della mobilità senza soffermarsi sul peso e senza conferire sufficiente autonomia alle determinanti di natura politica, sottovalutando così le molteplici configurazioni di potere dalle quali discendono scelte vincolanti sul ruolo dello stato produttore, gestore, regolatore del settore pubblico. La propugnata autonomia delle teorie fondate su explanans politici risulta però affievolita, poiché si limita a considerare l'intervento di attori, risorse, regole rilevanti nel processo decisionale secondo una prospettiva sistemica di carattere gerarchico, in forza della quale sono gli assetti stabili della politics a fissare confini e contenuti delle politiche. Essa privilegia un percorso esplicativo unilineare del policy making: delinea flussi e carichi decisionali determinati dalle esigenze funzionali inscritte nel circuito della rappresentanza democratica, ossia, in ultima analisi, dal livello di responsiveness del sistema politico.

Più specificamente, lo schema che rappresenta i seguenti stadi sequenziali

crescita delle domande $\rightarrow$ crescita delle funzioni $\rightarrow$ crescita (qualitativa e quantitativa) degli output $\rightarrow$ eventuali crisi da sovraccarico del sistema

traccia una ipotesi causale che non sembra spiegare esaustivamente i percorsi di sviluppo della politica pubblica, poiché si limita a considerare la complessa evoluzione genetico-istituzionale della policy come effetto congiunto di variazioni degli stimoli ambientali. Lasciando però irrisolto un problema esplicativo fondamentale: perché una politica pubblica persiste nel tempo anche se inefficace $o$, al contrario, viene modificata radicalmente sebbene non sussistano evidenti scollamenti fra gli output decisionali (programmi di investimento, regolamenti, leggi ad boc, ecc.), i provvedimenti implementati (quantità e qualità del trasporto pubblico) e le domande di mobilità, espresse o latenti, 
dei cittadini? A dar credito a queste teorie sfugge, insomma, una parte consistente delle variabili esplicative inerenti la politicità della politica pubblica, intesa come complesso dei fattori che sostanziano gli output prodotti dotandoli di legittimità e garantiscono, nel tempo, la cooperazione e la lealtà della comunità politica alle decisioni e ai programmi assunti.

Il superamento della impasse esplicativa implica un ripensamento del tema in questione - le mutevoli caratteristiche dell'intervento pubblico nel sistema dei trasporti - prendendo in considerazione anche altre variabili di natura politica e istituzionale. L'insieme dei fattori, cioè, che tendono a influenzare i processi decisionali nei diversi contesti nazionali e ai diversi livelli territoriali agendo «da dentro» alla politica pubblica. Vale a dire gli assetti stabili di funzioni, strutture e codici comportamentali tipicamente e specificamente condizionanti le scelte pubbliche su problemi di mobilità fisica. Assumiamo perciò che il rapporto fra variabili macro-economiche, politics e policy, lungi dal risolversi in una relazione gerarchica, si articola attraverso strutture e dinamiche reciprocamente influenti. Se nell'ambito della politics vengono formalizzate decisioni, programmi, regolamenti, diritti e spettanze, questi atti debbono tuttavia scontare la congruenza o meno ai principi normativi, ai valori e alle risorse scambiate dagli attori entro il campo d'azione organizzativo della politica pubblica.

\section{Mutamento e continuità nel policy making}

Come abbiamo accennato in riferimento al contesto euro-atlantico, le politiche dei trasporti hanno subito, nell'ultimo ventennio, una serie di cambiamenti che coinvolgono sia i mezzi e gli strumenti a disposizione degli attori rilevanti, sia le regole di interazione, sia i valori-obiettivi che legittimano le decisioni da adottares. Come altri settori economici nello stesso periodo, quello dei trasporti ha visto quindi mutare, con differenti pecu-

5 Hanno cioè subìto un tipo di mutamento che Peter Hall (1993) definisce di «terzo ordine». Sono cambiamenti di «primo ordine» gli aggiustamenti incrementali di alcuni strumenti della politica pubblica, in un quadro generale che vede però sostanzialmente inalterato l'insieme degli strumenti e degli obiettivi. I cambiamenti di «secondo ordine» consistono invece nella revisione completa degli strumenti della politica pubblica, senza alterarne gli obiettivi di fondo. 
liarità nazionali, da valutare caso per caso, il paradigma su cui poggia l'intero edificio della politica pubblica. Si è verificato altresì un altrettanto rimarchevole cambiamento nei loci d'autorità della policy, con l'ingresso di attori afferenti prevalentemente al mercato dei beni privati e alle istituzioni accademiche, ai quali è spesso assegnato il compito di legittimare l'insieme delle trasformazioni in atto. Sotto la pressione di vincoli ineludibili al risanamento dei conti pubblici, il tema delle inefficienze gestionali delle aziende controllate dallo stato si è tradotto, in gran parte dei paesi appartenenti alla Unione europea, nelle «ricette» di liberalizzazione e/o privatizzazione dei trasporti collettivi. È vero tuttavia che mutamenti di policy ampi, radicali e generalizzati come quelli intervenuti nel settore dei trasporti a partire dalla fine degli anni ' 70 non si giocano esclusivamente sul piano tecnico-scientifico, ma dipendono dalla configurazione globale dell'arena di potere entro la quale vengono prodotte le decisioni autoritative. A tale riguardo, la letteratura più accreditata sul policy change ha prevalentemente spiegato il mutamento come l'esito dello scontro fra differenti coalizioni, ciascuna portatrice di un proprio paradigma, a seguito di anomalie e fallimenti delle politiche perseguite. Cosicché il processo di cambiamento ha termine quando i supporters-fautori di uno dei paradigmi in competizione acquisiscono ruoli di autorità nel policy making, e sono pertanto in grado di modificare l'organizzazione e le procedure del processo politico, così come di istituzionalizzare il paradigma vincente (Hall 1993; Richardson et al. 1992; Sabatier e Jenkins Smith 1993).

Alcuni studi di caso condotti nell'ultimo decennio sul policy making dei trasporti non paiono confutare questa ipotesi, malgrado individuino altri caratteri del mutamento settoriale, che integrano, in parte, il modello sopra descritto. Seppur riconoscendo la rilevanza di molteplici fattori esogeni nel processo di generazione delle alternative, la tendenza all'innovazione o all'inerzia viene fatta risalire da questi autori alle caratteristiche strutturali delle reti di attori che si confrontano all'interno del variegato campo di analisi comunemente ricondotto al sistema dei trasporti. Ove, tuttavia, l'attributo della «sistemicità» è intrinsecamente sfidato dalla frammentazione dei diversi comparti modali (su strada, su ferro, marittimi e aerei), nonché dalla loro tendenza a creare reti decisionali specializzate, segmentate e scarsamente comunicanti fra loro (Kingdon 1984, 124 e ss.). Ciò nonostante, l'analisi di Dudley e Richardson (1996) sulle 
politiche dei trasporti stradali nel Regno Unito è lucida nel dimostrare che policy communities stabili non producono, come a lungo si è sostenuto, politiche pubbliche altrettanto stabili nel tempo, e quindi tendenzialmente immutabili a fronte degli accadimenti ambientali e delle pressioni esogene (Jordan e Richardson 1987). Al contrario, le dinamiche avversariali instauratesi fra i due reticoli decisionali dei trasporti stradali - quello degli interessi industriali, legato alle burocrazie del Ministero dei Trasporti, e quello degli interessi ambientali, connesso al mondo accademico ed al movimento «verde» - generano una sorta di rincorsa all'innovazione, allo scopo di controllare e depotenziare le posizioni di preminenza assunte da ciascuna configurazione decisionale nelle differenti arene di competizione da questi colonizzate. Tale spinta innovativa si verifica pertanto nel rispetto di almeno tre condizioni, implicitamente individuate dagli autori.

Innanzitutto, l'instaurazione e la strutturazione di reticoli organizzativi contrapposti tanto negli obiettivi da perseguire (da un lato la realizzazione di nuove infrastrutture per la viabilità, dall'altro la soppressione di questi programmi) quanto nei valori che sostengono i rispettivi schemi di azione (il sostegno allo sviluppo economico contro la difesa dei beni ambientali). In secondo luogo, la capacità da parte degli adversarial policy networks di «conquistare» accessi favorevoli presso una o più arene istituzionali. Se la lobby degli interessi motoristici ha goduto di legami privilegiati con il Ministero dei Trasporti, luogo istituzionalmente deputato alla elaborazione dei programmi di intervento, la policy community ambientalista ha potuto contrapporsi efficacemente allorché all'approccio nimby si è sostituita una strategia di respiro nazionale incanalata entro le procedure della Public Inquiry. In terzo luogo, l'esistenza entro ciascun policy network di comunità epistemiche e/o advocacy coalitions, ossia di attori in grado di generare consenso e legittimazione attorno a problemi e programmi in virtù delle loro risorse conoscitive, della loro capacità di superare barriere tecnologiche e di omogeneizzare i linguaggi specialistici con il «discorso comune». In entrambi i casi, sia per la rete interorganizzativa degli interessi industriali, sia per quella degli interessi ambientali, l'attivismo «imprenditoriale» di comunità epistemiche e advocacy coalitions è risultato un fattore determinante nel configurare tattiche e strategie di confronto, oltreché nel formulare le alternative di policy da sottoporre all'attenzione del legislatore e al giudizio del cittadino-elettore. 
L'esito di questa contrapposizione fra due reti conflittuali è la creazione di un sistema di contrappesi per il bilanciamento della rispettive sfere d'influenza. Cosicché la «rincorsa all'innovazione» di cui si parlava innanzi si risolve da un lato nella stabilità del settore, sotto l'azione dei controlli reciproci via via sperimentati dalle due policy communities, e dall'altro nel mutamento di strumenti e di micro-obiettivi decisionali, in un quadro competitivo che vede continui tentativi di acquisire vantaggi strategici dalle parti in lizza. Si tende dunque a dar luogo a dinamiche di policy change sebbene in presenza di una sostanziale stabilità nel novero degli attori in gioco. Si tratta tuttavia di processi di mutamento limitati a variazioni di primo e secondo ordine - per usare la scala di Hall (1993) - che non intaccano l'adesione a modelli di interazione ben consolidati nella loro struttura normativa, valoriale e relazionale. Eventuali mutamenti di terzo ordine, quelli che interessano il paradigma generale della politica pubblica, possono intervenire, secondo Dudley e Richardson (1996), sotto la spinta di ineludibili pressioni esogene, capaci di modificare l'ammontare delle risorse disponibili, oppure di offrire nuove opportunità tecnologiche per lo sviluppo dei sistemi di mobilità. Dipenderebbe pertanto dall'intervento di variabili esterne ai policy networks l'eventuale abbandono di strategie avversariali e la ricerca di un governo consensuale della mobilità, nell'ambito di un paradigma nuovo e condiviso. In assenza di queste pressioni esogene difficilmente si può pervenire ad un mutamento degli attuali equilibri, talché, in definitiva, ci possiamo aspettare una continuità nelle strategie di controllo reciproco attraverso l'occupazione di differenti arene ai diversi livelli di governo (locale, nazionale, e sovranazionale), oltre alla riproposizione dell'immagine avversariale come caratteristica chiave di questo particolare processo di policy (Dudley e Richardson 1996, 81).

Analoga, nella sostanza, la conclusione di Dunn (1995) circa l'evoluzione della politica autostradale in Francia. Anche in questo caso l'analisi delle interazioni evidenzia la netta contrapposizione fra due policy networks antagonisti, così come l'esito decisionale è costituito da una serie di cambiamenti incrementali che non incidono però sulla conservazione dei principi generali attorno ai quali si impernia l'azione di governo della policy. Ciò che invece cambia sensibilmente rispetto al caso britannico è l'insieme delle risorse strategiche a disposizione dei reticoli, ove si riscontra un accumulo sensibile di «vantaggi tattici» da 
parte del network costituito dalle burocrazie ministeriali (Ministeri dei Trasporti, delle Infrastrutture e delle Finanze) e dalla lobby autostradale nei confronti del network formato dai gruppi ambientalisti, da alcune amministrazioni locali e dalle organizzazioni degli interessi agricoli. Le dinamiche di mutamento non sono quindi conseguenti alla competizione fra due reticoli decisionali in equilibrio di forze, ma sono piuttosto il prodotto di un'azione anticipativa da parte di burocrazie tecniche e gruppi industriali copiosamente dotati di competenze tecnologiche e provvisti altresì di un'ampia riserva di sostegno presso l'opinione pubblica. In questo modo, il «vantaggio tattico» esibito dal «network autostradale» si traduce, all'atto pratico, sia in un dinamismo di policy capace di anticipare la domanda sociale, sia in un meccanismo inibitore nei confronti del network avverso, che non riesce ad elevare l'azione di protesta dalla dimensione nimby alle arene del confronto istituzionale. Si concretizza inoltre in una sorta di freno a modificazioni di più ampio respiro, attraverso le quali si possa sovvertire il monopolio statale nella fiscalizzazione dei carburanti e nella gestione dei pedaggi autostradali, il cui gettito costituisce una delle voci fondamentali della finanza pubblica francese.

Sebbene simile nelle sue dinamiche innovative, nella struttura delle configurazioni decisionali e nello stile avversariale delle loro relazioni, il settore ferroviario presenta in Francia differenti obiettivi di sviluppo. Come è esaustivamente descritto da Dunn e Perl (1994) nella loro analisi comparata dei processi di innovazione ferroviaria in Francia e Germania, la progettazione e la messa in opera del sistema ad alta velocità si colloca fra le strategie anticipative di un network stato-centrico assai prossimo, nella sua morfologia interorganizzativa, a quello del confinante network autostradale. È tuttavia conseguente ad un disegno di policy che pone al centro dei propri obiettivi la rivitalizzazione di alcuni fra i maggiori gruppi industriali transalpini, legati da rapporti di tipo simbiotico con le agenzie burocratiche del settore e fortemente penalizzati dalla crisi che ha investito le ferrovie occidentali a partire dagli anni ' 70 . Affrontare, dunque, con una politica di investimenti tecnologici l'inesorabile declino ferroviario è la risposta anticipativa della rete interorganizzativa, che ha affidato alle dinamiche del mutamento tecnologico la prospettiva di salvaguardia di un consistente settore occupazionale (quello dell'industria elettromeccanica) senza trovare resistenza significativa da parte del network avverso. Si tratta di 
una scelta strategica compiuta anche dalle ferrovie tedesche, all'interno di un contesto economico-produttivo che riproduce, a grandi linee, quello francese, tranne che per un rilevante aspetto interorganizzativo: il tipo di relazioni fra gli attori del policy network ferroviario è ascrivibile al modello corporativo, ove prevale l'inclusività e la contrattazione fra i soggetti coinvolti anziché la separazione e il confinamento entro campi avversariali. Sotto questo profilo, il policy change tecnologico appare come il risultato della mediazione istituzionalizzata fra le parti sociali. Ovvero come un classico esercizio di responsiveness nei confronti di più bisogni difficilmente conciliabili: il sostegno all'occupazione, l'efficacia del servizio, l'efficienza gestionale e la limitazione delle esternalità negative. Il tutto senza mettere in discussione i tratti costitutivi del governo settoriale, cioè senza modificare i principi paradigmatici entro i quali la politica pubblica è incardinata.

È superfluo asserire che gli studi sul policy making dei trasporti qui analizzati abbiano prodotto una serie di rilievi empirici assai significativi per sgomberare il campo dalla conventional wisdom che vuole il mutamento della politica pubblica come un attributo esclusivo della instabilità e della fluidità interorganizzativa. Essi hanno altresì generato ipotesi esplicative meritevoli di ulteriori approfondimenti: da un lato, alcuni contributi hanno posto l'accento sulla possibilità di concepire il mutamento come una strategia utile a mantenere in equilibrio $\mathrm{i}$ rapporti di forza fra reti interorganizzative fortemente stabilizzate e altrettanto fortemente contrapposte. Dall'altro lato, vi è chi rileva inequivocabili tendenze all'innovazione e al cambiamento anche laddove la prevalenza di policy networks stato-centrici abbia prodotto vantaggi tattici persistenti a favore di una specifica costellazione di attori, oppure abbia favorito la strutturazione di arene di mediazione in cui far confluire gli interessi confliggenti; in quest'ultima fattispecie, il cambiamento consegue alla capacità anticipativa del policy network rispetto alle pressioni esercitate dalla domanda sociale. In entrambi i casi, tuttavia, il fatto di riconoscere la rilevanza di variabili endogene nei processi di mutamento della politica dei trasporti sembra limitare la propria forza esplicativa a quegli aspetti del cambiamento che riguardano le componenti strumentali della politica pubblica, siano esse tecnologie, imposizioni regolamentari o innovazioni organizzative. Non paiono emergere, insomma, ipotesi interpretative che diano conto di quei cambiamenti nei prin- 
cipi, nei valori, negli orientamenti generali, oltreché negli strumenti di policy, che sono l'autentico fenomeno pervasivo del sistema dei trasporti europei da oltre un decennio a questa parte. Talché occorre ritornare all'ipotesi di Hall - corredata dalle integrazioni che le analisi del policy making hanno suggerito - per stabilire se ed in quale misura essa è in grado di spiegare i processi di cambiamento «di terzo grado» del settore dei trasporti.

Una prima, sommaria, ricognizione comparativa sembra tuttavia generare seri dubbi circa la capacità esplicativa di tale generalizzazione. I processi di mutamento intervenuti negli ultimi vent'anni configurano, con le opportune differenziazioni, autentici cambi di paradigma, ma, in gran parte dei casi, paiono affermarsi in contesti decisionali entro $i$ quali non si osserva una marcata conflittualità fra due o più coalizioni, ciascuna portatrice di uno specifico paradigma. Detto altrimenti, più che dal confronto fra costellazioni di attori dotati di valori-obiettivi ben declinati e contrapposti, la vittoria del paradigma neo-liberista sembra avere origine, nella maggior parte dei casi, dentro coalizioni dominanti stabilizzate. In tale contesto, il cambio di paradigma non è il frutto di una sfida da parte di attori in lotta per accedere alle posizioni di dominio istituzionale, ma l'esito di un «cambiamento di rotta» per aggiustamenti successivi, spesso portato a compimento dalla medesima coalizione o, talvolta, iniziato da una coalizione e terminato da una parte avversa senza stravolgerne i contenuti. Si può pertanto affermare che la liberalizzazione dei trasporti avviene, in prevalenza, entro arene relativamente stabili e per effetto di trasformazioni incrementali, più che di conflitti fra alternative concorrenti ${ }^{6}$. Questo sembra essere il cammino percorso da Francia, Germania, Svezia, Belgio, Olanda e Austria. Al contrario, hanno origine da un conflitto fra attori che sostengono paradigmi in competizione le politiche di privatizzazione dei trasporti messe in atto nel Regno Unito. Fra gli esempi di mancato compimento di veri e propri cambiamenti paradigmatici, con la commistione di obiettivi, mezzi e strumen-

6 La medesima tendenza è stata osservata da Coleman, Skogstad e Atkinson (1997, 274-275) nel campo delle politiche agricole. All'interno di tale settore «la transizione è molto più graduale e non è necessario che il mutamento istituzionale preceda quello di policy. $\grave{E}$ invece necessaria una distribuzione del potere nelle istituzioni tale da consentire agli attori la percezione delle conseguenze delle politiche pubbliche e di identificare paradigmi di policy alternativi. L'apprendimento di questo tipo induce alla negoziazione e al mutamento pianificato, che in definitiva costituisce un salto paradigmatico». 
ti incoerenti, quando non apertamente contraddittori, va invece annoverato il caso italiano. Come si può dunque concettualizzare il modello di «mutamento nella continuità» sperimentato da gran parte dei paesi europei? E perché, all'interno di tale continuità, in alcuni contesti il mutamento di paradigma pare più netto e in altri più sfumato, fino a risultare del tutto ineffettivo, o comunque confinato al terreno delle politiche simboliche, come nel nostro paese? Perché, infine, il caso inglese si accosta al modello conflittuale di policy change, piuttosto che a quello europeo-continentale? L'analisi dei processi di istituzionalizzazione delle politiche pubbliche offre un rilevante sostegno argomentativo per esprimere alcune ipotesi al proposito.

\section{L'istituzionalizzazione delle politiche}

Le politiche pubbliche attraversano processi di istituzionalizzazione e de-istituzionalizzazione dai quali dipende in larga misura la loro capacità di stabilire corsi d'azione vincolanti erga omnes. Con il termine istituzionalizzazione viene qui sintetizzato un insieme di attributi concernenti due dimensioni fondamentali della politica pubblica: quella morfologico-organizzativa e quella autoritativo-relazionale. La prima dimensione riguarda le caratteristiche del campo organizzativo della policy (Di Maggio $1983 ; 1986)$, con particolare riferimento alla struttura della coalizione dominante e delle barriere poste a presidio dell'ingresso nel campo (gatekeeping). Primario requisito formale è dunque la strutturazione di un campo organizzativo composto da organizzazioni, regole e procedure specificamente ascrivibili ai processi di decisione, o non decisione, in materia di mobilità fisica. A questo proposito, il campo organizzativo delle politiche dei trasporti presenta generalmente una articolazione al cui vertice risiede almeno un core actor istituzionale ${ }^{7}$. Sotto il profilo organizzativo-formale le politiche relative alla costruzione, gestione e sfruttamento commerciale di beni e servizi di trasporto si presentano pertanto in forma strutturata, e le ragioni

7 Il concetto di core actor istituzionale designa un attore dotato di almeno due attributi fondamentali: il possesso di status pubblico, politico e/o burocratico (generalmente legato all'assolvimento di una funzione ministeriale con facoltà di spesa); il fatto che a tale status facciano capo le risorse legali necessarie per programmare e coordinare gli interventi nella politica considerata. 
sono intuitive: la natura di bene pubblico o semi-pubblico tipica di questi sistemi e di queste infrastrutture, la ingente produzione di esternalità, il fatto che esse configurino spesso dei monopoli naturali e, più banalmente, che necessitino di ingenti investimenti con tempi di ammortamento assai lunghi, hanno condotto gran parte degli analisti a descrivere processi decisionali nei quali, salvo pochissime eccezioni, le decisioni chiave sono prese all'interno del circuito politico-amministrativo.

Si tratta di un caso particolarmente interessante per verificare il valore euristico delle ipotesi qui formulate. Ad un'osservazione superficiale, infatti, potrebbe sembrare che la politica dei trasporti si esaurisca nell'insieme di attività compiute dallo stato in materia di mobilità fisica, passeggeri e merci, con ciò implicitamente affermando la possibilità di procedere ad un'analisi di tipo struttural-funzionale. In realtà, il fatto che sia invariabilmente presente un core actor come sopra definito non significa affatto che esso sia necessariamente l'unico, e neppure il principale, responsabile della definizione del problema di policy, prima ancora che della scelta della soluzione. Elemento fondamentale per la strutturazione del campo organizzativo è la presenza di una coalizione dominante, formata dall'insieme degli attori che riescono a mobilitare, internamente alla configurazione interorganizzativa ed esternamente, nei confronti dei cittadini, consenso e legittimazione intorno alla propria leadership e al contenuto delle proprie azioni-decisioni. In altri termini, si tratta dei soggetti in grado di convogliare e coniugare interessi potenzialmente conflittuali e logiche d'azione eterogenee, generando giochi a somma positiva fra gli attori del campo, sotto forma di programmi e alternative di scelta giudicati autorevoli o convenienti. Con ciò, non è detto, quindi, che la funzione pivotale sia svolta da uno o più core actors istituzionali. $\grave{E}$ anzi frequente, nel campo dei trasporti, che il compito di riuscire a ottenere il consenso degli attori indifferenti o svantaggiati e di mantenere in tensione per il tempo necessario la rete di relazioni sia svolto da attori extra-istituzionali dotati o di particolari risorse tecnico-conoscitive (esperti, comunità epistemiche, opinion leaders) o di ingenti risorse finanziarie (gruppi industriali, istituti di credito).

Discende da quanto detto che la legittimazione della coalizione dominante dipende essenzialmente dal possesso, dalla esibizione, dal riconoscimento e dalla disponibilità all'uso di risorse-chiave per la generazione di decisioni vincolanti. Ove la 
«crucialità» delle risorse è da porre in relazione sia al tipo di ambiente istituzionale (tecnologico-strumentale o cerimonialeistituzionale) entro il quale avvengono prevalentemente gli scambi fra gli attori del campo, sia alla capacità della coalizione di esercitare effettivo dominio, ossia di controllare la struttura organizzativa, il patrimonio cognitivo ed epistemico delle altre organizzazioni, senza essere condizionata da altri attori attraverso processi isomorfici ${ }^{8}$. Utilizzando questa lente concettuale, si potrà quindi verificare l'applicabilità al settore dei trasporti delle metafore o immagini del potere che enfatizzano il ruolo dominante di specifici attori nel policy making nazionale: le burocrazie nel pantouflage francese; la diade governo-sindacati nel neo-corporativismo scandinavo e tedesco; la triangolazione imprese, burocrazia, commissioni parlamentari negli iron triangles statunitensi; i partiti politici nel party government all'italiana.

Come accennato, il processo di strutturazione del campo organizzativo comporta inoltre l'innalzamento di barriere all'ingresso (gatekeeping), volte a controllare la possibilità di co-determinare gli esiti del policy making da parte di attori esterni alla coalizione dominante. Per meglio inquadrarlo sotto l'aspetto empirico, il gatekeeping può essere inteso come l'insieme degli standard tecnici, economici e organizzativi ai quali occorre attenersi per influenzare le decisioni. Questi, nei trasporti, hanno generalmente veste giuridico-regolamentare (la legislazione protezionistica, antitrust, norme di sicurezza e di omologazione); burocratico-procedurale (commissioni, strutture amministrative ad hoc, conferenze dei servizi); ideologico-normativa (ad esempio, l'affermazione del droit de transport in Francia, oppure il consolidamento di sub-culture tecnologiche). Così definita, la misura del gatekeeping costituisce quindi un indicatore del livello di impermeabilità della coalizione dominante rispetto all'ambiente esterno (le altre sfere istituzionali e gli altri campi di policy) e all'ambiente interno (il contesto normativo della politica pubblica). Va detto, a questo proposito, che la morfologia e la consistenza del gatekeeping dipendono strettamente dalle «sfide» a cui debbono rispondere i decision makers, cioè dalla quantità e qualità di risorse dell'ambiente istituzionale prevalen-

$8 \mathrm{Ci}$ riferiamo qui in particolare ai tipi di isomorfismo mimetico e normativo, mediante $\mathrm{i}$ quali attori che monopolizzano la produzione delle conoscenze scientifiche (centri accademici, istituti di ricerca, reticoli di esperti) possono influenzare il processo di formulazione e attuazione delle politiche pubbliche, anche in presenza di istituzioni politiche fortemente istituzionalizzate e neutrali (Lanzalaco 1995, 197). 
te all'interno del campo organizzativo. Cosicché, in presenza di ambienti tecnologico-strumentali, le barriere tenderanno a salvaguardare il possesso di requisiti tecnici e conoscenze specialistiche, la capacità di stabilire sistemi di controllo e di coordinamento efficienti, la possibilità di instaurare processi decisionali improntati al rational problem solving. La funzione di filtro in ambienti cerimoniali-istituzionali si rivolge invece agli agenti in grado di influenzare la legittimità di regole, procedure, miti razionali, dal cui rispetto dipende l'efficacia della policy.

Il secondo fattore di istituzionalizzazione a cui facciamo riferimento concerne la forza decisionale della politica pubblica, qui intesa come risultante di due aspetti della produzione politica: uno, di natura formale, riguarda la tipologia delle decisioni collettive prodotte, classificate in base alla qualità dell'output; se, cioè, la policy regola gli interessi della collettività, ne privilegia alcune categorie, o piuttosto concorre a redistribuire sotto forma di servizi universalmente accessibili una fetta della ricchezza nazionale. L'altro aspetto, di natura sostanziale, concerne la forza vincolante dell'output nei confronti degli attori extra-istituzionali, vale a dire la capacità di generare cooperazione o conformità garantita, comportamenti leali, anche se gli esiti sono inefficaci per alcuni gruppi sociali o inefficienti sotto il profilo economico e tecnologico-strumentale. La creazione di ordine e cooperazione si basa infatti sulla capacità di offrire soluzioni tecniche, economiche ed organizzative giudicate imparziali rispetto alla struttura delle disuguaglianze presente nella società. E ciò si verifica allorché la coalizione dominante si dimostra in grado di controllare e filtrare il contesto normativo della politica pubblica, regolando efficacemente gli interessi delle forze sociali. In questo senso, le decisioni prodotte in contesti inter-organizzativi altamente istituzionalizzati «forniscono un insieme di regole, di procedure che regolano l'obbedienza, di norme di comportamento etico e morale che schermano dall'influenza dell'ambiente, modificano le motivazioni individuali, regolano il comportamento motivato dall'interesse personale $\mathrm{e}$ creano ordine e significato» (Olsen 1989, 13). Ipotizziamo pertanto che quanto maggiore la stabilità, la coesione, la specializzazione funzionale, la congruenza fra mappe cognitive nella coalizione dominante, in particolare fra «chi decide» e «chi mette in opera», il livello di strutturazione e di impermeabilità del gatekeeping (attributi della dimensione morfologico-organizzativa), l'universalismo delle decisioni emesse e la probabilità che le azioni programmate siano realizzate con efficacia e rac- 
colgano consenso sociale (attributi della dimensione autoritativo-relazionale), tanto più elevato risulti il grado di istituzionalizzazione della politica pubblica.

\section{Istituzionalizzazione, continuità e mutamento}

Passando ora ad analizzare le dinamiche evolutive che caratterizzano le politiche dei trasporti, cerchiamo di spiegare perché l'alto grado di istituzionalizzazione agisce come un potente fattore di sviluppo endogeno del campo organizzativo, che tende a creare strategie di protezione e difesa rispetto ai fattori di mutamento esogeno. Innovazioni tecnologiche, shock economici, cambiamenti repentini dello scenario internazionale, mutamenti a livello di politics e di policy (nuove maggioranze di governo, modificazioni delle regole del gioco istituzionale), costituiscono potenziali elementi di discontinuità nelle scelte di poli$c y$. La misura dell'influenza esercitata è tuttavia connessa alla resistenza e al filtro che gli attori del campo organizzativo frappongono alle pressioni esogene. Per mezzo di quali meccanismi il grado di istituzionalizzazione della policy può incrementare o abbassare la capacità di difesa del campo di azione organizzativo - e, al suo interno, della coalizione dominante - dalle dinamiche esogene? Come agisce sulle capacità di policy change $\mathrm{e}$ come, invece, sulle attitudini alla policy inertia? A questo proposito si possono ipotizzare tre scenari idealtipici:

1) È probabile che la politica pubblica resti tanto più indefinita negli obiettivi e nei propositi quanto minore il suo grado di istituzionalizzazione. In contesti a bassa istituzionalizzazione, la presenza nel campo di azione organizzativo di una pluralità di principi di legittimazione, di forme e di paradigmi organizzativi, di relazioni sociali, fa sì che mutamenti esogeni provochino alterazioni frequenti e improvvise nel set di risorse e nella posta in gioco, quindi nei rapporti di forza fra gli attori del network. La vulnerabilità delle coalizioni dominanti rispetto agli agenti ambientali e il basso livello di gatekeeping tendono a generare politiche frammentarie, disorganiche, i cui processi decisionali e di implementazione sono penetrati e condizionati da specifiche costellazioni di gruppi miranti all'appropriazione di risorse disaggregate. La bassa istituzionalizzazione non consente di garantire la conformità generalizzata delle forze sociali alle decisioni assunte; da qui il ricorso anche frequente a forme di protesta, boicottaggio o inosservanza delle regole. Lo sviluppo del- 
la politica dei trasporti risulta essere, in questo caso, dipendente dalle risorse dei gruppi portatori degli interessi economici «forti» (l'industria automobilistica, ferrotranviaria, autotrasportistica), dalle capacità organizzative di categorie ristrette (ferrotranvieri, piloti e controllori di volo, autotrasportatori) e dalla capacità di mobilitazione degli interessi diffusi (gruppi ambientalisti) presenti nel settore, dalle relazioni di conflitto/alleanza fra questi attori instaurate, dal tipo di rapporti che li lega alla burocrazia. Principali fonti di autorevolezza delle decisioni sono i giudizi di esperti e opinion-makers, che tendono a monopolizzare il ruolo strategico di creatori e mediatori del consenso. La policy si risolve nella produzione di decisioni subsettoriali o microsezionali, con scambi di breve periodo e di livello meso o micro-economico.

2) E inoltre probabile che il passaggio ad obiettivi diversi, e quindi la formulazione di politiche di ampio raggio, quali le riforme settoriali, si verifichi se il grado di istituzionalizzazione della policy risulta elevato. Come abbiamo detto, l'istituzionalizzazione rende i risultati della politica (output e outcome) relativamente indipendenti dalle modificazioni ambientali; ma, d'altro lato, ciò non impedisce una reattività fisiologica a mutamenti delle variabili di tipo economico e politico (altre sfere istituzionali, altre politiche pubbliche). In contesti ad elevata istituzionalizzazione emergono infatti le capacità di filtro, di interpretazione, di tuning, di adeguamento costante dei tempi di risposta o di non-risposta grazie al controllo di mappe cognitive e patrimoni epistemici da parte degli attori istituzionali. Pressioni sociali, economiche e politiche subiscono così processi di reinterpretazione e riconfigurazione che possono portare o alla riformulazione di procedure già sperimentate o allo sviluppo di un nuovo ciclo di policy. Di conseguenza, è assai più frequente il verificarsi di politiche concertate, che regolano ampie categorie di interessi, il cui orizzonte temporale è di medio-lungo periodo e $\mathrm{i}$ cui effetti intenzionali sono di portata macro-economica. Le conseguenze allocative, sia di natura redistributiva, sia di tipo selettivo, non comportano forme oppositive extraistituzionali, dato l'alto grado di legittimità della coalizione dominante. L'elevata istituzionalizzazione consente di ottenere alti livelli di loyalty allorché inserisce i potenziali oppositori entro un sistema di relazioni strutturali, che contemplano vincoli procedurali e ideologico-normativi. Quando, cioè, eroga simboli di identità e produce forme di appartenenza che si estrinsecano in vere $\mathrm{e}$ proprie sub-culture di policy. Nel settore dei trasporti tali con- 
dizioni possono verificarsi nei contesti in cui, oltre a sancire un diritto sociale al trasporto, si è provveduto a integrare gruppi e movimenti oppositivi entro un processo di pianificazione della mobilità e del territorio. Laddove si tende quindi a dar luogo ad uno stile di policy improntato al problem solving, in virtù del quale si sviluppa «un orientamento all'interesse comune, ossia valori o norme che sono distinti dall'auto-interesse individuale dei partecipanti e che, perciò, possono facilitare l'accordo volontario anche quando sono necessari sacrifici in termini di interessi individuali» (Scharpf 1989, 261).

3) Il livello elevato della istituzionalizzazione può tuttavia avere ripercussioni opposte sullo sviluppo della policy. È infatti possibile che campi di policy iper-istituzionalizzati creino le premesse per il verificarsi di una sorta di freezing bypotesis della politica pubblica, il cui effetto principale è l'impedimento di forme di adattamento all'ambiente. In questo caso, le barriere e gli agenti della istituzionalizzazione, ossia le «specifiche configurazioni di organizzazioni e di azioni in grado di strutturare, modellare e influenzare tanto i processi economici che le classi o i gruppi di interesse» (Meny e Thoenig 1991, 95), producono schermature, depotenziamento e routinizzazione degli interessi esogeni, al punto di replicare per inerzia il medesimo modello di policy. L'eccesso di legittimazione può indebolire, fino a eliminarlo del tutto, il legame fisiologico fra domande e livello di responsiveness, sviluppando così un circuito decisionale autoreferenziale. Il collasso di politiche dei trasporti consolidate e fortemente legittimate si può quindi imputare $a$ incapacità ricettive di ciò che viene elaborato in ambienti interni (sviluppo di nuove tecnologie, sviluppo di nuove forme di coordinamento) o esterni (impatto di altre politiche, ad esempio quelle energetiche, o di crisi del sistema economico/produttivo).

Condizioni e caratteristiche del mutamento in prospettiva comparata

I dati relativi allo sviluppo delle politiche dei trasporti in cinque democrazie euro-atlantiche ${ }^{9}$ (Regno Unito, Francia, Sve-

9 L'analisi comparata condotta in questo paragrafo costituisce la sintesi di uno studio più ampio e circostanziato (Tebaldi 1999) al quale rimandiamo per ulteriori approfondimenti. 
zia, Italia e Stati Uniti) ci permettono innanzitutto di osservare che all'aumento del grado di istituzionalizzazione le politiche adottate assumono tratti connotativi più nitidi; il paradigma di policy tende insomma a proporre con maggiore «purezza» le caratteristiche dei modelli idealtipici lungo l'asse stato-mercato. Ciò si verifica in tutti i paesi in cui le politiche dei trasporti hanno subito processi di istituzionalizzazione conclamati. Ove, cioè, i campi organizzativi presentano i requisiti di stabilità, coesione, specializzazione funzionale, congruenza nelle mappe cognitive di policy makers e implementors, alte barriere all'ingresso dei policy networks. E, sotto il profilo della qualità dell'output, le decisioni assunte risultano tendenzialmente universalistiche e dotate di livelli elevati di responsiveness ed efficacia.

Nel Regno Unito, a partire dal secondo dopoguerra, i mutamenti di paradigma delle politiche dei trasporti sono stati radicali, e sono intervenuti all'indomani di altrettanto radicali cambiamenti a livello di maggioranze governative. Cosicché le politiche di nazionalizzazione prima, e di privatizzazione poi, potrebbero sembrare la diretta emanazione degli esiti elettorali che hanno condotto al potere i laburisti nel 1945 e 1964, e i conservatori nel 1979. E, tuttavia, l'analisi diacronica mostra che, in entrambi i casi, il mutamento di paradigma non è il risultato di rivolgimenti tanto radicali quanto repentini delle coalizioni dominanti della politica pubblica, in seguito ad uno scontro fra sostenitori di opposti paradigmi. Il cambiamento di obiettivi e strumenti in senso «statalista» trova la sua piena attuazione a partire dagli anni ' 50 , ma si configura come l'atto conclusivo di un lungo processo di nazionalizzazione dei trasporti cominciato venti anni prima e giunto a compimento per passi successivi, trasversalmente alle diverse maggioranze di governo.

In modo non dissimile, la riforma in senso privatistico dei governi conservatori è il frutto di aggiustamenti successivi entro una coalizione dominante della politica pubblica relativamente stabile, che inizia a esibire il progetto durante il primo mandato Thatcher, per poi vederlo concretamente realizzato quattordici anni dopo, con le prime vendite di linee ferroviarie gestite dal governo Major. Vendite che sono proseguite anche nel primo periodo di governo del leader laburista Blair, nel rispetto del programma di privatizzazione messo in atto a suo tempo dai conservatori. L'analisi del processo di privatizzazione ferroviaria dimostra inoltre che la conflittualità inter-coalizionale concerne 
prevalentemente strategie «di facciata», ossia il campo delle politiche simboliche, mentre il mutamento di paradigma è il prodotto di contrattazioni e conflitti intra-coalizionali, all'interno di una coalizione sostanzialmente omogenea e coesa.

Le politiche dei trasporti svedesi anticipano, rispetto a quelle del Regno Unito, il loro processo di istituzionalizzazione, tanto che dai primi anni ' 30 si assiste alla strutturazione del modello di monopolio pubblico, che proseguirà fino alla metà degli anni '70. Anche in questo contesto, la relativa stabilità e persistenza della coalizione di governo a guida socialdemocratica non è, di per sé, l'elemento esplicativo fondamentale per comprendere l'evoluzione della policy. Al di là della stabilità di governo, occorre sottolineare che le principali spinte al cambiamento di tipo paradigmatico sono sopravvenute, fin dalla prima metà degli anni '80, all'interno della medesima coalizione dominante della politica pubblica, e sono state legittimate, a livello di politics, tanto dai governi conservatori, quanto da quelli socialdemocratici. Il caso svedese evidenzia inoltre che alla iperistituzionalizzazione della politica si è effettivamente accompagnata, nell'ultima fase di sviluppo, una maggiore propensione ad accogliere obiettivi e a proporre soluzioni che rimandano alla competizione pianificata, pur permanendo evidenti caratteri di monopolio pubblico. In altri termini, la spinta al cambiamento ha dato luogo a formule ibride, che non paiono maturare, a più di un ventennio dal loro avvio, una impostazione univoca del paradigma di policy.

La persistenza di forti poteri monopolistici in capo alle burocrazie pubbliche convive con aperture parziali dei mercati dei trasporti, all'interno di rigidi schemi programmatici, come dimostra la recente evoluzione del settore ferroviario. L'approccio al mercato, sebbene dichiarato nei documenti di pianificazione strategica e nei «libri bianchi» delle agenzie burocratiche, trova evidenti sbarramenti di natura organizzativa. Questi sono, in primo luogo, dovuti all'altezza delle barriere ideologico-normative poste a presidio del network decisionale, che impediscono il pieno accesso al principio della libera concorrenza fra operatori privati; in secondo luogo, alla peculiare vischiosità del policy process svedese, che trova nel sistema delle commissioni un luogo istituzionale in cui la negoziazione viene proceduralizzata e segregata, fino ad assottigliare o, talvolta, azzerare la percezione dei flussi di feedback.

Il caso francese è di più complessa lettura. La progressiva 
istituzionalizzazione della politica pubblica, culminata nelle scelte monopolistiche degli anni ' 80 , ha interessato prevalentemente il trasporto ferroviario e quello urbano/metropolitano, ove si è registrata una evidente propensione al potenziamento dei servizi, al miglioramento delle performances e al controllo delle esternalità. Al vertice della configurazione decisionale si osserva il rafforzamento dei legami fra ministero dei trasporti, agenzie tecnico-burocratiche e maggiori imprese private del settore ferrotranviario. Si struttura fra questi attori un rapporto simbiotico i cui vincoli, oltreché dagli interessi convergenti, sono rafforzati dalla adesione e interiorizzazione di valori comuni. Tali valori sono prevalentemente di natura tecnologico-organizzativa e fanno capo ad un «modello originario» che vede in posizione pivotale le alte burocrazie ministeriali. La centralità e la stabilità dei ruoli burocratici sono da ascrivere al possesso di almeno tre risorse strategiche, che legittimano l'azione della coalizione dominante e il contenuto delle politiche: l'expertise in materie tecnico-economiche; il prestigio sociale e professionale; la fitta rete di rapporti informali, che si estende sia in ambito burocratico, sia nel mondo dell'impresa pubblica e privata. Quest'ultima fattispecie, in particolare, permette ai vertici amministrativi di agire in qualità di brokers o imprenditori di policy elevando l'efficacia del sistema decisionale. Essa deriva dai caratteri organizzativi su cui è imperniato sia il reclutamento amministrativo, sia il controllo delle politiche dei trasporti.

Il primo carattere concerne la socializzazione professionale delle più alte cariche dello stato (politiche e burocratiche) e dei vertici aziendali (privati e nazionalizzati). Entrambe le categorie provengono infatti, in grande maggioranza, dalle Grandes Écoles tecniche, dalle quali ricevono orientamenti, valori e mappe cognitive comuni, che facilitano i rapporti fra «chi decide» e «chi mette in opera» la politica pubblica, e che agiscono trasversalmente, nei processi decisionali, alle affiliazioni partiticoideologiche. Il secondo carattere è di natura interorganizzativa e riguarda la frequenza con cui ricorre il fenomeno del pantouflage, ossia il passaggio dei funzionari di più alto grado dai grandi corpi burocratici alla guida delle maggiori imprese, e viceversa. Ciò indica in modo sufficientemente nitido una elevata capacità di presidiare continuativamente le barriere all'ingresso (gatekeeping) della coalizione dominante. In ragione di tale stabilità e impermeabilità del policy network, oltreché degli alti indici di efficacia delle politiche perseguite (in particolare l'alta 
velocità ferroviaria e il trasporto pubblico della capitale), il settore ferrotranviario sta vivendo, da oltre 40 anni, un periodo di sostanziale continuità nelle linee paradigmatiche e negli strumenti impiegati. Tanto che il modello di monopolio pubblico, contrariamente alle altre esperienze europee esaminate, non è stato sfidato da paradigmi alternativi, né a livello centrale, né a livello locale.

La complessità del caso francese nasce tuttavia dall'esame di altri sub-settori di policy, segnatamente quello automobilistico e dell'autotrasporto (merci e collettivo). Qui la vigenza delle regole di mercato si è generalmente accompagnata a interazioni collusive fra gli attori del network (come nel caso delle politiche automobilistiche, fra Ministero del Tesoro, aziende autostradali a partecipazione pubblica, industrie di costruzioni infrastrutturali e industria dell'automobile), oppure a veri e propri oligopoli privati (così nel trasporto collettivo su gomma). Talché gli aspetti regolativi, benché rilevanti, risultano secondari rispetto agli ampi spazi di manovra concessi agli operatori privati attraverso provvedimenti marcatamente micro-sezionali. Ne consegue una politica pubblica a due facce: una in via di crescente istituzionalizzazione e solidamente ancorata ad un modello di sviluppo che pone al centro del paradigma dominante il rispetto del trasporto come bene pubblico, secondo il principio del droit de transport, almeno limitatamente al sub-settore ferrotranviario. L'altra, debolmente istituzionalizzata, descrive percorsi paradigmatici più tortuosi; se, da un lato, richiama le regole della concorrenza, dall'altro interviene per depotenziarle con finalità e strumenti distributivi.

Nell'ambito dei casi a debole istituzionalizzazione, quello italiano sembra corroborare, più di altri, la nostra ipotesi. In quasi 150 anni di storia dei trasporti esso presenta una evidente propensione alla atomizzazione dei poteri politico-amministrativi, entro un modello fondato sull'equilibrio fra due obiettivi: quello del monopolio privato nel settore automobilistico e quello della collusione distributiva nei trasporti pubblici collettivi. Sorprende anzi la stabilità di tale modello bicefalo anche sotto la pressione di accadimenti esogeni al campo organizzativo della politica pubblica, sia di natura socio-economica, sia di matrice politico-istituzionale. Il basso grado di istituzionalizzazione sembra essere, dunque, un potente fattore di continuità del paradigma di policy (rectius della commistione fra diversi paradigmi di policy). Da un lato, l'inconsistenza delle barriere all'in- 
gresso del circuito decisionale rende possibile la rappresentanza di una miriade di interessi legati, a vario titolo, allo sviluppo dei differenti sub-settori (stradale, ferroviario, urbano, aereo). Dall'altro lato, gli interventi normativi volti a conferire elementi connotativi più marcati al paradigma di policy, sia in senso statalista, sia in direzione privatistica, hanno sempre mostrato limiti evidenti all'atto della loro implementazione.

Ripercorrendo a grandi linee le vicende regolative dei trasporti nel nostro paese, fin dall'esordio i provvedimenti di nazionalizzazione ferroviaria si configurano così non tanto come una strategia di gestione coordinata e unitaria delle reti, in vista della necessaria modernizzazione del servizio, quanto come una gigantesca operazione di salvataggio degli esercenti privati, che coincidono con i maggiori gruppi finanziari italo-tedeschi. Successivamente, quando già il successo dell'automobile faceva emergere rivalità e sovrapposizioni fra i modi di trasporto privato e i servizi collettivi, le politiche dei trasporti pubblici in ambito urbano e suburbano non sono state integrate da misure restrittive del trasporto privato su gomma che redistribuissero in forma equa le esternalità da questo prodotte. Inoltre, gli enti municipalizzati del trasporto urbano quasi mai hanno agito secondo regole di trasparenza ed efficienza amministrativa, ed hanno anzi contribuito all'instaurazione di rapporti collusivi con aziende private (o semi-pubbliche) fornitrici di beni infrastrutturali e di materiali rotabili. Infine, l'indirizzo privatistico enunciato da molteplici provvedimenti all'inizio degli anni ' 90 , sembra anch'esso restare nelle intenzioni del legislatore. Recano infatti segnali del tutto discordanti con tale principio le difficoltà incontrate nel processo di trasformazione dell'ente ferroviario di stato (Fs) in società per azioni, con regime proprietario limitato alle sole infrastrutture. Così come desta non pochi sospetti l'ambiguità della formula di project financing dell'alta velocità, visto che la partecipazione degli imprenditori privati ai rischi di investimento risulta ampiamente garantita dal capitale pubblico. Né sembra mutare significativamente il quadro consolidato delle regole del gioco l'intervento della normativa comunitaria sulla liberalizzazione dei trasporti pubblici e sulla apertura internazionale delle relative gare di appalto. Del tutto funzionale ai meccanismi di «penetrazione» dei poteri pubblici da parte di attori appartenenti alla sfera economica è la latitanza, a tutti i livelli di governo, di strumenti di pianificazione intermodale grazie ai quali concepire unitariamente non solo le decisioni sulle 
grandi infrastrutture (i maggiori assi di comunicazione) ma soprattutto le strategie regolative, tecnico-organizzative, commerciali e finanziarie relative ai diversi modi di trasporto e al loro riequilibrio sul medio-lungo periodo. Cosicché anche l'approccio della concorrenzialità intermodale, frequentemente evocato da esperti e tecnici di settore come volàno di efficienza ed economicità, concorre a «congelare» l'attuale assetto di gestione separata dei diversi mondi trasportistici, trascurando, per contro, le opzioni di complementarietà.

Il caso statunitense presenta anch'esso bassi livelli di istituzionalizzazione nei primi tre periodi evolutivi della politica. Alla elevata permeabilità del campo organizzativo nei confronti dei grandi interessi industriali fa riscontro sia lo sviluppo di relazioni tutt'altro che avversariali fra poteri pubblici e imprese tanto a livello federale, quanto a livello statale e locale - sia un'applicazione controversa di forme regolative antitrust volte a disciplinare i rapporti economico-commerciali del settore. $\mathrm{Ne}$ consegue che la «fede neoclassica» nei meccanismi di mercato viene spesso abbandonata per far posto a modelli di governo in cui le autorità pubbliche, più che il ruolo di garante, intervengono con provvedimenti distributivi alterando le regole della concorrenza. A cominciare dalle sovvenzioni concesse dai governi statali per la costruzione prima, e la manutenzione poi, delle linee ferroviarie nel primo periodo di sviluppo dei trasporti di massa; favorendo, quindi, lo sviluppo dell'industria automobilistica con interventi diretti (tagli ai finanziamenti per il trasporto pubblico urbano) e indiretti (costruzione capillare di strade e autostrade prive di pedaggi). In entrambi i casi viene sensibilmente attenuata la capacità regolativa pubblica del mercato (seppur unica, rispetto ai paesi europei, per estensione e complessità) attuando giochi collusivi fra attori pubblici e privati sia nel momento decisionale, sia nella fase di messa in opera.

Fino alla fine degli anni '60 la politica dei trasporti statunitense è dunque caratterizzata da indicatori di bassa istituzionalizzazione e dalla convivenza di orientamenti interventisti con principi liberisti, all'interno di un modello tipicamente clientelare. Spiccano, in questo senso, la forte capacità partecipativa degli interessi industriali in tutte le fasi del policy making, una certa propensione cooperativa fra decisore pubblico e capitale privato, la tendenziale emarginazione dei soggetti portatori di interessi diffusi dai loci decisionali, la frammentazione di questi 
ultimi, con prevalenza dei livelli statale e locale di intervento rispetto al livello federale, l'assenza di forme coordinate di pianificazione. Si tratta tuttavia di un quadro che ha subito rilevanti modificazioni a partire dai primi anni ' 70 , in direzione di una crescente istituzionalizzazione della politica pubblica. Se già dal 1964 si tende a dar luogo a politiche di finanziamento federali volte a favorire lo sviluppo di sistemi integrati di trasporto collettivo nelle maggiori aree metropolitane, la linea interventista diventa più consistente durante gli anni ' 70 , in seguito alla forte opposizione sociale che contestava i privilegi concessi al trasporto privato (anti-freeway revolt).

Nonostante i problemi di bilancio e l'indirizzo marcatamente liberista degli anni ' 80 abbiano provocato un sensibile contenimento dell'intervento federale nel finanziamento del trasporto pubblico, si è tuttavia assistito, a partire dagli anni ' 90 , ad un sostanziale riequilibrio della politica dei trasporti, ad una maggiore apertura e trasparenza dei mercati, al proliferare di iniziative miranti al potenziamento delle reti urbane e suburbane (in particolare su ferro) secondo i requisiti della economicità di esercizio, della co-partecipazione al rischio gestionale fra attori pubblici e investitori privati, della programmazione pluriennale nella definizione delle scelte prioritarie. Il tutto collocato all'interno di un quadro normativo organico e definito anche negli aspetti operativi, oltreché tecnico-finanziari, che disciplina unitariamente problematiche trasportistiche, ambientali e territoriali secondo comuni indirizzi programmatici.

\section{Conclusione}

Pochi dubbi sussistono sulla salienza dei processi di mutamento quale chiave esplicativa dei percorsi evolutivi attraversati dalle politiche pubbliche. All'interno di questo problema di ricerca, si accrescono quantitativamente e, soprattutto, qualitativamente gli studi ${ }^{10}$ che - riprendendo una linea argomentativa già tracciata da Lowi $(1964 ; 1972)$ e cercando di sfuggire alla banalizzazione dei rapporti fra politics e policy - considerano i percorsi di sviluppo delle politiche pubbliche come variabili in-

10 Fra i più recenti contributi di taglio teorico e comparativo, si vedano i lavori di Knoke et al. (1996); Coleman, Skogstad e Atkinson (1997); Pierson (1993; 1996). 
dipendenti dalle determinanti esogene correntemente evocate per spiegarne l'evoluzione nei regimi poliarchici (le altre dimensioni dell'agire sociale ed economico o le relazioni di potere inscritte nella competizione per i ruoli di autorità). Supposto che tali fattori influenzino la policy come variabili intervenienti, occorre pertanto verificare tempi, modi e misure con cui esse vengono interpretate, riconfigurate, schermate, depotenziate, routinizzate dalle barriere e dagli agenti della istituzionalizzazione. Questa ipotesi di lavoro può aprire nuovi spazi interpretativi all'analisi dei mutamenti paradigmatici che hanno interessato le politiche dei trasporti dei maggiori paesi europeo-occidentali nel corso dell'ultimo ventennio. Mutamenti che difficilmente possono essere spiegati nelle loro differenti articolazioni facendo esclusivo ricorso alle categorie concettuali e agli schemi comparativi tradizionalmente impiegati dalle scienze politologiche nel settore dei trasporti.

Il ragionamento vale tanto per le politiche di privatizzazione dei trasporti collettivi implementate nel Regno Unito, quanto per le soluzioni ibride adottate negli altri contesti, sia quelle più orientate alla liberalizzazione dei mercati (Svezia), sia quelle più inclini a mantenere un sostanziale controllo pubblico di alcuni settori e di privatizzarne altri (Francia e Germania). Ovvero, per tutti quei casi in cui mutamenti di portata radicale, seppur variabili per estensione dei modi di trasporto regolati, siano spesso condivisi da maggioranze bipartisan, da categorie di interessi eterogenei, da ampi settori della società civile e dell'opinione pubblica, indipendentemente dalle fluttuazioni dei cicli economici. Casi, insomma, che sfuggono a letture incentrate esclusivamente su fattori di politics o su variabili economiche, e che neppure possono essere ricondotti in toto alle argomentazioni esibite dalla letteratura più accreditata sul policy making settoriale, ove il mutamento viene interpretato come l'esito dello scontro fra differenti coalizioni, a seguito di fallimenti o anomalie di politiche antecedenti.

Le dinamiche organizzative e autoritativo-relazionali che si legano ai processi di istituzionalizzazione delle politiche pubbliche consentono, a nostro avviso, di mettere a fuoco con maggiore precisione interpretativa proprio quei caratteri evolutivi del governo della mobilità altrimenti sfuggenti all'indagine empirica. In primo luogo, esse permettono di concettualizzare il modello di mutamento nella continuità sperimentato da gran parte dei paesi europei. Vale a dire, di emendare quelle spiega- 
zioni, fatte proprie anche dagli approcci neo-istituzionali, secondo cui i processi di strutturazione e stabilizzazione dei policy networks vanno prevalentemente coniugati alla staticità e all'inerzia dei processi di produzione politica.

In secondo luogo, chiariscono perché, all'interno di tale modello dinamico, in alcuni contesti il mutamento di paradigma appare più netto $\mathrm{e}$ in altri più sfumato, fino a risultare del tutto ineffettivo, o comunque confinato al terreno delle politiche simboliche, come nel nostro paese. Il passaggio dalla nazionalizzazione alla liberalizzazione dei trasporti ha avuto piena effettività ed efficacia laddove (Svezia, Regno Unito) coalizioni dominanti stabili e coese sono dotate di un alto grado di specializzazione funzionale, di mappe tecnologico-cognitive congruenti fra «chi decide» e «chi mette in opera», di solide barriere all'ingresso dei policy networks, oltreché di un certo grado di responsiveness delle decisioni emesse. Al contrario, malgrado l'inserimento pressoché contemporaneo della issue nell'agenda politica, il processo di liberalizzazione dei trasporti collettivi trova, in un contesto a bassa istituzionalizzazione come quello italiano, grandi difficoltà di implementazione. In questo caso - a dispetto della sostanziale stabilità della configurazione decisionale ascrivibile alla politica dei trasporti - la frammentazione dei campi organizzativi, insieme alla scarsa dotazione di risorse cognitive da parte delle coalizioni dominanti, alla permeabilità nei confronti degli interessi organizzati e alla ineffettività dei piani di sviluppo settoriale, costituiscono fattori inerziali di elevata forza frenante per qualsivoglia cambiamento paradigmatico.

In terzo luogo, infine, l'alto grado di istituzionalizzazione della politica pubblica dei trasporti chiarisce perché e a quali condizioni il caso britannico possa essere accostato ad un modello tendenzialmente avversariale di policy change, come alcuni osservatori tendono a sottolineare, e malgrado ciò produca cambiamenti paradigmatici, contraddicendo quegli stessi osservatori, anche in assenza di spinte esogene dirompenti.

\section{Riferimenti bibliografici}

Button, K.J. (1990), Environmental Externalities and Transport Policy, in «Oxford Review of Economic Policy», n. 2, pp. 61-75.

Coleman, W.D., G.D. Skogstad e M.M. Atkinson (1997), Paradigm Shifts and Policy Networks: Cumulative Change in Agriculture, in «Journal of Public Policy», n. 3, pp. 273-301. 
Dilorenzo, T.J. (1983), Economic Competition and Political Competition: An Empirical Note, in «Public Choice», n. 2, pp. 203-209.

Di Maggio, P.J. (1983), State Expansion and Organizational Fields, in R.H. Hall e R.E. Quinn (a cura di), Organizational Theory and Public Policy, Beverly Hills, Sage, pp. 147-163.

- (1986), Structural Analysis of Organizational Fields. A Blockmodel Approach, in L.L. Cummings e B. Staw (a cura di), Research in Organizational Behavior, Greenwich, JAI Press, vol. 8, pp. 335370.

Dudley, G. e J.J. Richardson (1996), Why Does Policy Change Over Time? Adversarial Policy Communities, Alternative Policy Arenas, and British Trunk Roads Policy, 1945-1995, in «Journal of European Public Policy», n. 1, pp. 63-83.

Dunn, J.A. (1995), The French Highway Lobby. A Case Study in StateSociety Relations and Policymaking, in «Comparative Politics», $\mathrm{n}$. 3, pp. 275-295.

Dunn, J.A. e A. Perl (1994), Policy Networks and Industrial Revitalization: High Speed Rail Initiatives in France and Germany, in «Journal of Public Policy», n. 3, pp. 311-343.

Dunn, W. (1981), Public Policy Analysis, Englewood Cliffs, Prentice Hall.

Etzioni, A. (1990), Policy Implications of Socio-Economics, in «Policy Studies Review», n. 3, pp. 445-454.

Fokkema, T. e P. Nijkamp (1994), The Changing Role of Governments: The End of Planning History?, in «International Journal of Transport Economics», n. 2, pp. 127-145.

Glaister, S. e C. Mulley (1983), Public Control of the British Bus Industry, Aldershot, Gower.

Hall, P.A. (1993), Policy Paradigms, Social Learning, and the State. The Case of Economic Policymaking in Britain, in «Comparative Politics», n. 3, pp. 275-296.

Jordan, A.G. e J.J. Richardson (1987), Government and Pressure Groups in Britain, Oxford, Clarendon Press.

Kingdon, J.W. (1984), Agendas, Alternatives and Public Policies, Boston, Little, Brown.

Knoke, D. et al. (1996), Comparing Policy Networks. Labor Politics in the U.S., Germany and Japan, Cambridge, Cambridge University Press.

Kohl, J. (1983), The Functional Structure of Public Expenditures. LongTerm Changes, in C.L. Taylor (a cura di), Why Governments Grow. Measuring Public Sector Size, Beverly Hills, Sage, pp. 201216.

Lanzalaco, L. (1995), Istituzioni, organizzazioni, potere, Roma, Nis. Lowi, T.J. (1964), American Business, Public Policy, Case-Studies, and Political Theory, in «World Politics», n. 4, pp. 677-715. 
- (1972), Four Systems of Policy, Politics and Choice, in «Public Administration Review», n. 4, pp. 298-310.

Mc Bride, M.E. (1990), The Economic Approach to Political Behavior: Governors, Bureaucrats, and Cost Commissions, in «Public Choice», n. 2, pp. 117-136.

Mc Guire, R.A. et al., (1987), The Determinants of the Choice between Public and Private Production of a Publicly Funded Service, in «Public Choice», n. 3, pp. 211-230.

Meny, Y. e J.-C. Thoenig (1991), Le politiche pubbliche, Bologna, Il Mulino.

Olsen, J. (1989), Scienza politica e teoria dell'organizzazione, in «Rivista Italiana di Scienza Politica», n. 1, pp. 3-22.

Pierson, P. (1993), When Effect Becomes Cause: Policy Feedback and Political Change, in «World Politics», n. 4, pp. 595-628.

- (1996), The New Politics of the Welfare State, in «World Politics», n. 2, pp. 143-179.

Pint, E.M. (1990), Nationalisation and Privatisation: A Rational Choice Perspective on Efficiency, in «Journal of Public Policy», n. 3, pp. 267-298.

Richardson, J.J. (1979), Policy Making and Rationality in Sweden: The Case of Transport, in «British Journal of Political Science», n. 3, pp. 341-353.

Richardson, J.J. et al. (1992), The Dynamics of Policy Change: Lobbying, and Water Privatization, in «Public Administration», n. 70, pp. 157-175.

Rokkan, S. (1982), Metodi e modelli nell'analisi comparata dei processi di costruzione della nazione, in S. Rokkan, Cittadini, elezioni, parti$t i$, Bologna, Il Mulino, pp. 95-129.

Rostow, W. (1971), Politics and the Stages of Growth, Cambridge, Cambridge University Press.

Sabatier, P. e H. Jenkins Smith (1993), The Dynamics of Policy Oriented Learning, in P. Sabatier e H. Jenkins Smith (a cura di), Policy Change and Learning, Boulder, Westview Press, pp. 41-55.

Scharpf, F. (1989), Decision Rules, Decision Styles and Policy Choices, in «Journal of Theoretical Politics», n. 2, pp. 149-176.

Stigler, G.J. (1971), The Theory of Economic Regulation, in «Bell Journal of Economics and Management Science», n. 2, pp. 114-141.

Tebaldi, M. (1999), La politica dei trasporti, Bologna, Il Mulino. 